\title{
New Abundances From Very Old Stars
}

\author{
Terese Hansen* \\ Landessternwarte, Heidelberg University \\ E-mail: thansen@lsw.uni-heidelberg.de \\ C. J. Hansen \\ Dark Cosmology Center, Copenhagen \\ E-mail: cjhansen@dark-cosmology.dk \\ N. Christlieb \\ Landessternwarte, Heidelberg University \\ E-mail: nchristliebalsw.uni-heidelberg.de

\section{Yong}

Research School of Astronomy and Astrophysics, The Australian National University, Weston, ACT 2611, Australia

E-mail: yong@mso.anu.edu.au

\section{T. C. Beers}

Department of Physics and JINA-CEE: Joint Institute for Nuclear Astrophysics - Center for the Evolution of the Elements, University of Notre Dame, Notre Dame, IN 46556, USA

E-mail: tbeers@nd.edu

\section{J. Andersen}

Niels Bohr Institute, University of Copenhagen

E-mail: jaeastro.ku.dk

Metal-poor stars provide the fossil record of Galactic chemical evolution and the nucleosynthesis processes that took place at the earliest times in the history of our Galaxy. From detailed abundance studies of low mass, extremely metal-poor stars $([\mathrm{Fe} / \mathrm{H}]<-3)$, we can trace and help constrain the formation processes which created the first heavy elements in our Galaxy. Here we present the results of a $\sim 25$-star homogeneously analysed sample of metal-poor candidates from the Hamburg/ESO survey. We have derived abundances for a large number of elements ranging from $\mathrm{Li}$ to $\mathrm{Ba}$, covering production processes from hydrostatic burning to neutron-capture. The sample includes some of the most metal-poor stars $([\mathrm{Fe} / \mathrm{H}]<-4)$ studied to date, and also a number of stars enhanced in carbon. The so called CEMP (carbon enhanced metal-poor) stars, these stars make up $\sim 20 \%$ of the stars with $[\mathrm{Fe} / \mathrm{H}]<-3$, and $80 \%$ of the stars with $[\mathrm{Fe} / \mathrm{H}]<-4.5$. The progenitors of CEMP stars are still not fully constrained; they could be a result of binary mass transfer or high-mass explosive events in the early universe.

XIII Nuclei in the Cosmos,

7-11 July, 2014

Debrecen, Hungary

\footnotetext{
* Speaker.
} 


\section{Introduction}

The elemental abundances of metal-poor stars (stars with $[\mathrm{Fe} / \mathrm{H}] \leq-1$ ) are widely recognized as being the footprint of the first stars to form in our Galaxy. The first stars were presumably very massive due to the lack of cooling agents, resulting in short lifetimes that ended in a supernova (SN) explosions, thereby enriching the inter-stellar medium (ISM) with elements heavier than helium. From recent studies of large samples of metal-poor stars [4, 14, 21] it has become evident that a large fraction of the metal-poor stars are carbon enhanced, the so-called CEMP stars. The CEMP stars can be divided into several sub-classes, depending on the level of neutroncapture element abundances they exhibit. The two main sub-classes of the CEMP stars are the CEMP-no and CEMP-s stars. The CEMP-no stars exhibit no over-abundances of neutron-capture elements, while the CEMP- $s$ stars exhibit over-abundances of neutron-capture elements produced by the slow neutron-capture process (s-process). CEMP-s stars are generally believed to be the product of mass transfer from a asymptotic giant-branch (AGB) star companion, which has now evolved into a white dwarf, a picture supported by radial-velocity monitoring [15, 8]. For the CEMP-no stars, however, the picture is less clear and several progenitors have been suggested for these stars, including pollution by faint $\mathrm{SNe}$ that experienced extensive mixing and fallback during their explosions $[27,28,25,26,12,13,20]$, winds from massive, rapidly rotating, mega metal-poor stars, also referred to as "spinstars" [10, 18, 11, 19, 6], or mass transfer from an AGB companion $[24,16]$.

\section{Sample}

We have analyzed $\sim 25$ metal-poor stars observed with the UVES/VLT spectrograph, with spectra having an average resolwing power of $R \sim 45000$ and signal-to-noise of 40 at $4000 \AA$. We performed a 1D LTE abundance analysis using the MOOG code with alpha-enhanced $([\alpha / \mathrm{Fe}]=$ $+0.4)$ Kurucz model atmospheres [5]. Effective temperatures was determined from the infrared flux method, gravities are from $\mathrm{Y}^{2}$ isochrones [7] assuming an age of $10 \mathrm{Gyr}$ and an alpha enhancement of $[\alpha / \mathrm{Fe}]=+0.3$, and metallicity was derived from Fe I lines. This analysis procedure was also used in the Most Metal-Poor Stars project of Yong et al. [29]. Combining the two datasets results in a total of over 200 homogeneously analyzed stars, a sample that includes some of the most metal-poor stars known today.

Table 1 list the number of stars found in the combined sample for a given metallicity, and the number of these stars which are carbon enhanced. As can be seen, the fraction of carbonenhanced stars rises from about half for stars with $[\mathrm{Fe} / \mathrm{H}]<-2$ to two thirds at $[\mathrm{Fe} / \mathrm{H}]<-4$. Table 2 shows the stars in the combined sample divided into the different sub-classes of metalpoor stars, NMP (for "normal" metal-poor), CEMP-no, CEMP- $s$, and CEMP-r/s, along with the original definition of the CEMP sub-classes from Beers \& Christlieb (2005) [2]. The majority of the known CEMP stars are of the CEMP-s variety, but it has been shown that at the lowest metallicities $([\mathrm{Fe} / \mathrm{H}]<-3.0)$ the CEMP-no stars are the dominant sub-class [1]. We find a roughly equal number of CEMP-no and CEMP-s stars in the combined sample, as expected since about half of the stars in the combined sample have $[\mathrm{Fe} / \mathrm{H}]<-3$, where the CEMP-no stars dominate. 


\begin{tabular}{l|r|r|r|r} 
& {$[\mathrm{Fe} / \mathrm{H}]<-4$} & {$[\mathrm{Fe} / \mathrm{H}]<-3$} & {$[\mathrm{Fe} / \mathrm{H}]<-2$} & {$[\mathrm{Fe} / \mathrm{H}]<-1$} \\
\hline Total & 12 & 102 & 208 & 213 \\
C-enhanced & 8 & 25 & 55 & 59
\end{tabular}

Table 1: Total number and number of carbon-enhanced stars in the combined sample at different metallicities.

\begin{tabular}{c|c|c|c|c} 
Star & NMP & CEMP-no & CEMP- $s$ & CEMP-r $/ s$ \\
\hline Def. & {$[\mathrm{Fe} / \mathrm{H}]<-1$} & {$[\mathrm{Fe} / \mathrm{H}]<-1$} & {$[\mathrm{Fe} / \mathrm{H}]<-1$} & {$[\mathrm{Fe} / \mathrm{H}]<-1$} \\
& & {$[\mathrm{C} / \mathrm{Fe}]>+0.7$} & {$[\mathrm{C} / \mathrm{Fe}]>+0.7$} & {$[\mathrm{C} / \mathrm{Fe}]>+0.7$} \\
& & {$[\mathrm{Ba} / \mathrm{Fe}]<+0.0$} & {$[\mathrm{Ba} / \mathrm{Fe}]>0.0$} & {$[\mathrm{Ba} / \mathrm{Fe}]>0.0$} \\
& & & {$[\mathrm{Ba} / \mathrm{Eu}]>+0.5$} & $0.0<[\mathrm{Ba} / \mathrm{Eu}]<+0.5$ \\
\hline$\#$ & 155 & 24 & 29 & 5
\end{tabular}

Table 2: Definition of the main sub-classes of CEMP stars, and the number of stars in each sub-class in the combined sample. Note that we employ $[\mathrm{C} / \mathrm{Fe}]>+0.7$ to define the CEMP stars, rather than the level of $[\mathrm{C} / \mathrm{Fe}]>+1.0$ as originally suggested by Beers \& Christlieb (2005).

\subsection{Abundances}

Figure 1 shows the derived abundances for the combined sample of $\mathrm{Ca}, \mathrm{Cr}$, and $\mathrm{Ba}$, examples of an alpha-element, iron group-element, and a neutron-capture element, as a function of metallicity. NMP stars are shown in black, CEMP-no stars in red, and CEMP-s stars in green. For the alpha-element a small over-abundance is seen, reflecting the enrichment from one or more corecollapse SNe; also a very low star-to-star scatter is seen for both the alpha- and iron-group elements. The abundances of the CEMP-no and CEMP- $s$ stars are indistinguishable from those of the NMP stars for these two groups of elements.

Examination of the bottom panel of Figure 1, where derived abundances of the neutron-capture element barium are shown, reveals a quite different picture. The Ba abundances exhibit a large scatter, indicating more than one production channel may apply for the neutron-capture elements. The CEMP- $s$ stars are clearly isolated from the rest due to the large over-abundances of Ba, but there is no clear separation between the Ba abundances of the NMP stars and the CEMP-no stars.

Lithium abundances in metal-poor stars was first explored in the work of Spite and Spite [23], who discovered the so-called Spite plateau, and concluded that the similar Li abundances for their metal-poor dwarfs, independent of metallicity, may represent the lithium produced by Big Bang nucleosynthesis. We detect lithium for two of the most metal-poor stars in our sample, both of which are ultra metal-poor CEMP-no stars, one with $[\mathrm{Fe} / \mathrm{H}]=-4.7$ and one with $[\mathrm{Fe} / \mathrm{H}]=-4.2$ [9]. Figure 2 shows the $\mathrm{Li}$ abundances of these two stars as a function of luminosity, along with $\mathrm{Li}$ abundances measured for other CEMP-no stars taken from Masseron et al. (2012) [17]. All of the CEMP-no stars have Li abundances below the Spite plateau, indicating that lithium has been depleted in these stars. For the giant stars, convection within the star itself can lead to Li depletion, but for the dwarfs the measured Li abundance should refect that of the ISM from which the star formed. The Li abundances in CEMP-no stars can be explained if the progenitors of the CEMP-no stars are massive stars, which could have destroyed their initial lithium due to internal burning. When this Li-astrated material mixes with the ISM, the CEMP-no stars that subsequently form 


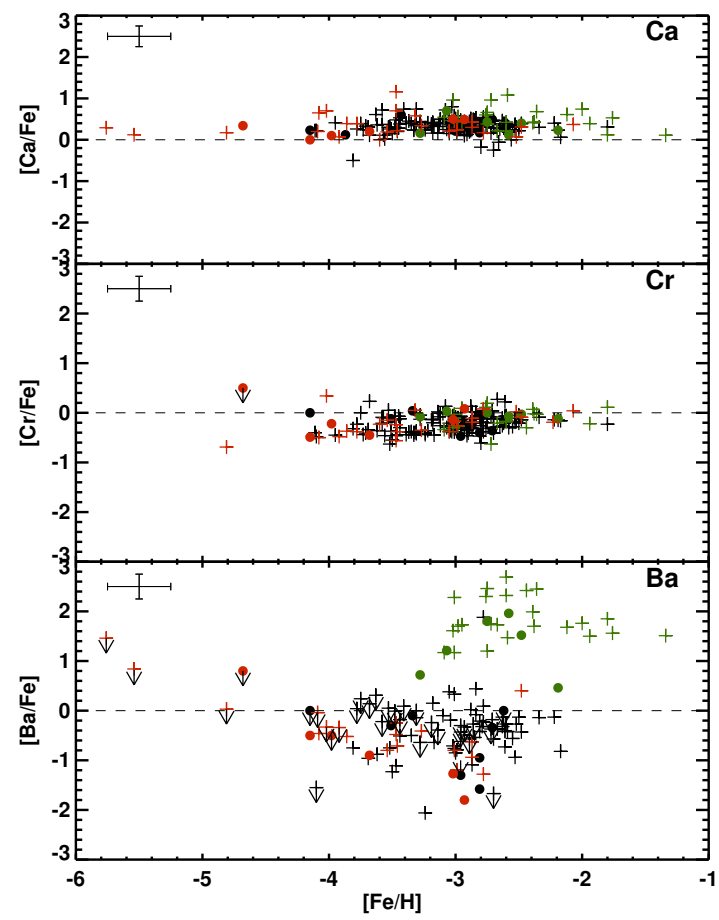

Figure 1: Derived abundances for $[\mathrm{Ca} / \mathrm{Fe}]$ (top), $[\mathrm{Cr} / \mathrm{Fe}]$ (middle), and $[\mathrm{Ba} / \mathrm{Fe}]$ (bottom), as a function of metallicity. The three types of metal-poor stars, NMP (black), CEMP-no (red), and CEMP-s (green) in the sample are shown. Dots are from Hansen et al. (2015). and the crosses are from Yong et al. (2013). Upper limits are indicated by arrows, and the dashed line is the solar value

would have overall lower Li abundances [19, 22].

\subsection{Binary properties}

To asses the binary properties of CEMP-no and CEMP-s stars, we have monitored a sample of 45 CEMP stars with the Nordic Optical Telescope for a period of $\sim 7$ years. The observations have resulted in a dataset of roughly 12 spectra per star. These have been cross-correlated against a template to detect any shift in the radial velocity over the seven years of monitoring. For bright targets, velocity shift down to $300 \mathrm{~ms}^{-1}$ can be detected. Twenty one stars in the sample are CEMPno stars, of which five show radial-velocity variation. Twelve stars in the sample are CEMP-s stars, of which ten show variation in their radial velocity over the seven years of monitoring.

\section{Summary}

We have presented the results of an abundance analysis of $\sim 25$ metal-poor stars, and for the radial-velocity monitoring of another 45 CEMP stars. At low metallicities a large fraction of the stars are carbon-enhanced. The two major groups are the CEMP- $s$ and CEMP-no stars; the CEMPno stars dominate at metallicities $[\mathrm{Fe} / \mathrm{H}] \leq-3.0$. We find abundances for the alpha elements 


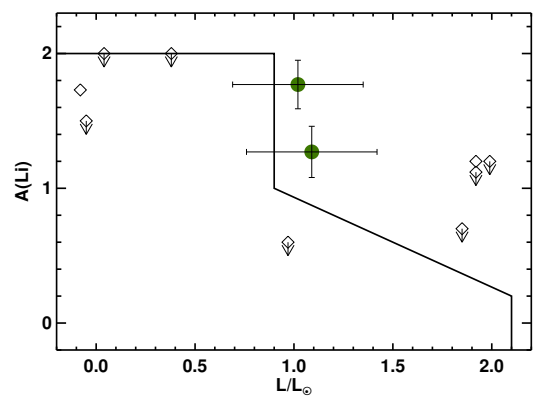

Figure 2: Lithium detections for two of the CEMP-no stars in the sample, the solid line shows the Spite plateau and the depletion due to stellar evolution [9].

and iron-peak elements in the CEMP- $s$ and CEMP-no stars that are indistinguishable from the non carbon enhanced stars in the sample. For the neutron-capture elements the CEMP-s stars are identified by their high Ba abundances, where as the CEMP-no and NMP stars have similar abundances. The lithium abundances found in CEMP-no stars are all below the Spite plateau, and are suggested to be due to lithium depletion due to burning in massive first stars and subsequent mixing with primordial gas prior to the formation of the CEMP-no stars. Results of radial-velocity monitoring demonstrates that, while the pollution from a binary companion is a good explanation for the abundance abnormalities seen in the CEMP- $s$ stars, as found in earlier work [15], it is not an acceptable explanation for the CEMP-no stars. So far both spinstars and mixing and fall back SNe are possible progenitors of the CEMP-no stars, but further analysis of larger samples of CEMP-no stars is needed to better constrain these possibilities.

\section{References}

[1] W. Aoki, Carbon-Enhanced Metal-Poor (CEMP) stars,IAU Symposium, 2010 (265) 111

[2] T.C. Beers \& N. Christlieb, The Discovery and Analysis of Very Metal-Poor Stars in the Galaxy, Nature 43531 (2005).

[3] D. Carollo et al., Two stellar components in the halo of the Milky Way, Nature, 4501020 (2007).

[4] D. Carollo at al., Carbon-enhanced Metal-poor Stars in the Inner and Outer Halo Components of the Milky Way, Astrophys. J., 744195 (2012).

[5] F. Castelli \& R.L Kurucz, New Grids of ATLAS9 Model Atmospheres, IAU Symposium 210 20P (2003).

[6] G. Cescutti at al., The s-process in the Galactic halo: the fifth signature of spinstars in the early Universe?, Astron. \& Astrophys., 553 A51 (2013).

[7] P. Demarque at al., YY Isochrones with an Improved Core Overshoot Treatment, Astrophys. J.S, 155 667 (2004).

[8] T. Hansen et al., Heavy Elements in the Early Galaxy, arXiv:1301.7208, (2013).

[9] T. Hansen et al., Exploring the Origin of Lithium, Carbon, Strontium, and Barium with Four New Ultra Metal-poor Stars, 787162 (2014). 
[10] R. Hirschi et al., Stellar evolution of massive stars at very low metallicities, Reviews in Modern Astronomy, 19 (2006).

[11] R. Hirschi, Very low-metallicity massive stars:. Pre-SN evolution models and primary nitrogen production, Astron. \& Astrophys., 461571 (2007).

[12] H. Ito et al., BD+44 493: A Ninth Magnitude Messenger from the Early Universe; Carbon Enhanced and Beryllium Poor, Astrophys. J.L., 698 L37 (2009).

[13] H. Ito et al., Chemical Analysis of the Ninth Magnitude Carbon-enhanced Metal-poor Star BD+44493, Astrophys. J., 77333 (2013).

[14] Y.S. Lee et al., Carbon-enhanced Metal-poor Stars in SDSS/SEGUE. I. Carbon Abundance Estimation and Frequency of CEMP Stars, AJ 146132 (2013).

[15] S. Lucatello et al., The Binary Frequency Among Carbon-enhanced, s-Process-rich, Metal-poor Stars, Astrophys. J., 625825 (2005).

[16] T. Masseron et al., A holistic approach to carbon-enhanced metal-poor stars, Astron. \& Astrophys., 509, A93, (2010)

[17] T. Masseron et al., Lithium Abundances in Carbon-enhanced Metal-poor Stars, Astrophys. J., 751, 14, (2012)

[18] G. Meynet et al., The early star generations: the dominant effect of rotation on the CNO yields, Astron. \& Astrophys., 447, 623, (2007)

[19] G. Meynet et al., Are C-rich ultra iron-poor stars also He-rich? Astron. \& Astrophys., 521, A30, (2010)

[20] K. Nomoto, C. Kobayashi \& N. Tominaga, Nucleosynthesis in Stars and the Chemical Enrichment of Galaxies, Annual Review of Astronomy and Astrophysics, 51, 457, (2013)

[21] J.E. Norris et al., The Most Metal-poor Stars. IV. The Two Populations with [Fe/H] <-3.0, Astrophys. J., 762, 28, (2013)

[22] L. Piau et al., From First Stars to the Spite Plateau: A Possible Reconciliation of Halo Stars Observations with Predictions from Big Bang Nucleosynthesis, Astrophys. J., 653, 300, (2006)

[23] F. Spite \& M. Spite, Abundance of lithium in unevolved halo stars and old disk stars - Interpretation and consequences Astron. \& Astrophys., 115, 357, (1982)

[24] T. Suda et al., Is HE 0107-5240 A Primordial Star? The Characteristics of Extremely Metal-Poor Carbon-Rich Stars, Astrophys. J., 611, 476, (2004)

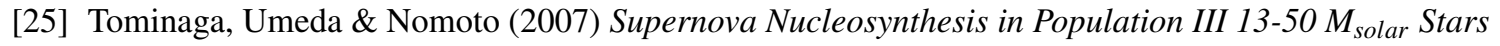
and Abundance Patterns of Extremely Metal-poor Stars, Astrophys. J., 660, 516, (2007)

[26] N. Tominaga, N. Iwamoto \& K. Nomoto, Abundance Profiling of Extremely Metal-poor Stars and Supernova Properties in the Early Universe, Astrophys. J., 785, 98, (2013)

[27] H. Umeda \& K. Nomoto, First-generation black-hole-forming supernovae and the metal abundance pattern of a very iron-poor star, Nature, 422, 871, (2003)

[28] H. Umeda \& K. Nomoto Variations in the Abundance Pattern of Extremely Metal-Poor Stars and Nucleosynthesis in Population III Supernovae, Astrophys. J., 619, 427, (2005)

[29] D. Yong et al., The Most Metal-poor Stars. II. Chemical Abundances of 190 Metal-poor Stars Including $10 \mathrm{New}$ Stars with $[\mathrm{Fe} / \mathrm{H}]<=-3.5$, Astrophys. J., 762, 26, (2013) 